\title{
Esterilización Femenina
}

\author{
Dres.: Carlos Alberto Esparza Duque* y Mario Calle Mesa** \\ UNIVERSIDAD DE CALDAS - FACULTAD DE MEDICINA \\ HOSPITAL UNIVERSITARIO DE CALDAS \\ DEPARTAMENTO DE OBSTETRICIA Y GINECOLOGIA
}

\section{INTRODUCCION}

El método anticonceptivo más utilizado en la actualidad es la esterilización voluntaria. La esterilización quirúrgica femenina es, a su turno, la modalidad más favorecida en todo el mundo, con excepción de escasos países. Entre las diferentes técnicas para efectuarla se destaca, sin duda, la esterilización laparoscópica. En este campo de aplicación, la endoscopia ginecológica con sus brillantes y acelerados progresos, ha logrado éxito sobresaliente (1).

El propósito de nuestrò estudio es evaluar la experiencia sobre esterilización femenina, en el Departamento de Obstetricia y Ginecología del Hospital Universitario de Caldas y compararla con la de otros autores.

\section{Material y Métodos}

Se estudian 460 pacientes esterilizadas por laparoscopia-electrocoagulación y 69 por minilaparotomía-Pomeroy, entre el 1o. de mayo de 1976 y el 31 de julio de 1978.

\footnotetext{
* Profesor Asociado

* Jefe del Departamento
}

Se establecieron los siguientes requisitos para admitir las pacientes al programa:

1. Mujer de 25 años cumplidos o más.

2. Tener 3 hijos vivos, por lo menos

3. Firmar, con el esposo o el compañero en casos de unión estable, una constancia de solicitud voluntaria del procedimiento.

Estos requisitos fueron modificados en casos especiales, como por ejemplo de pacientes con graves trastornos psiquiátricos $u$ otros problemas médicos serios.

Para la laparoscopia se empleó la técnica de una sola punción subumbilical con electrocoagulación y extirpación de un pequeño fragmento de trompa en la mayoría de los casos. En algunas pacientes sólo se realizó electrocoagulación y sección, sin extirpar el segmento tubario. El sistema es de tipo monopolar, de alta frecuencia.

Para minilaparotomía se siguió el procedimiento descrito por Vitoon Osathanond (2) y un equipo especial que cuenta con elevador uterino, gancho tubario, separadores y otros instrumentos de uso 
general en cirugía. La esterilización se hizo por la técnica de Pomeroy (3).

Ambas técnicas de esterilización se emplearon como programa ambulatorio, con observación post-operatoria de unas pocas horas.

A todas las pacientes se les dio una cita de control a los 7 días, para evaluación de los efectos inmediatos de la intervención.

La información fue registrada en formularios precodificados, del tipo empleado por el Programa Internacional de Investigaciones en Fecundidad (IFRP) y por la Corporación Centro Regionalde Población (CCRP) en el Programa Regional de Investigaciones en Fecundidad, PRIF con algunas modificaciones.

Los datos se procesaron en computador. Para el cálculo de las estadísticas se eliminaron los datos faltantes.

\section{Resultados}

Tabla No. 1

\section{DISTRIBUCION SEGUN AREA} DE RESIDENCIA

\begin{tabular}{lrc}
\hline \multicolumn{1}{c}{ Area } & Número & Porcentaje \\
\hline Urbana & 367 & 69.4 \\
Urbana fuera del área & 47 & 8.9 \\
Rural & 64 & 12.1 \\
Rural fuera del área & 51 & 9.6 \\
\hline Total & 529 & 100.0 \\
\hline
\end{tabular}

Se observa que el $78.3 \%$ corresponde a la ciudad de Manizales $(69.4 \%)$ y aotras zonas urbanas $(8.9 \%)$.
Tabla No. 2

DISTRIBUCION POR EDADES

\begin{tabular}{lrr}
\hline Grupos de Edad & Número & Porcentaje \\
\hline 24 años o menos & 2 & 0.4 \\
$25-29$ años & 138 & 26.1 \\
$30-34$ años & 173 & 32.7 \\
$35-39$ años & 149 & 28.2 \\
$40-44$ años & 64 & 12.1 \\
45 años o más & 3 & 0.6 \\
\hline Total & 529 & 100.0 \\
\hline
\end{tabular}

La edad promedio fue de 33.3 años, con un rango de 21 a 45 .

Tabla No. 3

NUMERO DE HIJOS VIVOS

\begin{tabular}{lcrcc}
\hline No. Hijos & Varones & Porcentaje & Mujeres & Porcentaje \\
\hline 0 & 23 & 4.4 & 32 & 6.1 \\
$1-2$ & 275 & 52.2 & 275 & 52.3 \\
$3-4$ & 171 & 32.4 & 153 & 29.1 \\
$5-6$ & 48 & 9.1 & 52 & 9.9 \\
$7-8$ o más & 10 & 1.9 & 14 & 2.7 \\
\hline Total & 527 & 100.0 & 526 & 100.0 \\
\hline
\end{tabular}

El promedio es de 2.5 tanto para varones como para mujeres. El $10.5 \%$ tienen hijos de un solo sexo. El número total de hijos varones y mujeres fue de 1.336 y 1.322 , respectivamente.

Casi la mitad de las pacientes $(48.5 \%)$, solicitaron la esterilización, cuando el hijo menor tenía menos de 1 año, es decir, relativamente pronto después de su último parto.

En cuanto al número de abortos previos, el $53 \%$ no había tenido ninguno. El promedio para las pacientes que sí habían tenido abortos fue de $1.9 \%$. 
Tabla No. 4

\section{ANTICONCEPCION PREVIA}

\begin{tabular}{lrc}
\hline Método & Número & Porcentaje \\
\hline Ninguno & 119 & 22.5 \\
DIU & 100 & 18.9 \\
Anticonceptivos orales & 196 & 37.0 \\
Esterilización previa & 4 & 0.8 \\
Condón & 25 & 4.7 \\
Ritmo/coito interrupto & 49 & 9.3 \\
Espuma/jalea/diafragma & 6 & 1.1 \\
Otro & 30 & 5.7 \\
\hline Total & 529 & 100.0 \\
\hline
\end{tabular}

El $38.6 \%$ de las pacientes o bien no utilizaba ningún método anticonceptivo $(22.5 \%)$, o empleaba algunos de los menos eficaces, como ritmo, jaleas, espumas, etc.

Cuatro pacientes con esterilización previa fueron sometidas nuevamente a laparoscopia o a minilaparotomía, por falla del procedimiento inicial.

Más de la mitad $(55.9 \%)$ estaba empleando anticonceptivos orales o dispositivos intrauterinos.

El estudio se refiere básicamente a pacientes del servicio general del hospital, ya que solamente $8.9 \%$ fueron de carácter privado.

El $95.1 \%$ fue manejado ambulatoriamente,

El peso promedio fue de $55.9 \cdot \mathrm{kg}$ con un rango de 32 a 85.

Solamente hubo 4 pacientes de 80 kg o más de peso.
En cuanto a cirugías previas, el $10.6 \%$ habían tenido alguna cirugía abdominal previa y el $7.2 \%$ alguna cirugía pélvica.

El $94.1 \%$ de las pacientes no había padecido enfermedad sistémica alguna de importancia.

Al practicar la laparoscopia o la minilaparotomía se encontró que el $80 \%$ tenía aparato genital normal. A las restantes se les encontró alguna patología como adherencias, miomas uterinos, quistes ováricos, varicocelex pélvico, etc.

Se consideró que había infección pélvica crónica en un $8.5 \%$ de los casos.

Tabla No. 5

ANESTESIA

\begin{tabular}{lcc}
\hline & Número & Porcentaje \\
\hline General & 110 & 20.8 \\
Analgesia + A. Local & 410 & 77.5 \\
Analegsia + A. Local & & \\
+ General & 6 & 1.1 \\
Otra & 3 & 0.6 \\
\hline Total & 529 & 100.0 \\
\hline
\end{tabular}

Se empleó analgesia y anestesia local en el $77.5 \%$ de los casos. Se administraron $10 \mathrm{mg}$ de diazepan I.V. y 1.3 a 1.5 . $\mathrm{mg} / \mathrm{kg}$ de meperidina I.V. La lidocaína se usó al $1 \%$. En 6 pacientes esta medicación fue insuficiente y se hizo necesario recurrir a la anestesia general para terminar la intervención. Se administró anestesia general a $20.8 \%$ de las pacientes. Sólo se usaron antibióticos postperatorios, excepcionalmente, en $5.3 \%$.

No se presentaron complicaciones del procedimiento (laparoscopia o minilaparotomía), ni de la cirugía (electrocoagulación o Pomeroy), en 91.3 y $94.4 \%$ de los casos respectivamente. 


\section{Comentario}

De los resultados precedentes se colige que nuestra zona de influencia en el programa de planificación familiar mediante esterilización femenina, es urbana casi en un 80 por ciento, concretamente la ciudad de Manizales.

Sería interesante confrontar los datos de este estudio en cuanto a edad de las usuarias (media de 33.3 años), con una revisión de años más recientes, a ver si se observa la tendencia anotada en algunos países a aceptar la esterilización a edades más jóvenes (1).

Encontramos un promedio de hijos vivos por mujer de 5 . Beck reporta un promedio de 2.9 , en 1979 (4-5). Como punto de comparación podemos citar 2.6 en Inglaterra (1975) y 8.7 en Sudán (1975).

Casi la mitad de las solicitantes de esterilización lo hizo cuando el último hijo tenía menos de 1 año. No podemos por el presente estudio determinar cuántas de ellas habían tenido fallas de métodos anticonceptivos, ni cuántas reconocían que habían ya sobrepasado el número de hijos deseados. La motivación para no tener más hijos perduró en muchas hasta un año después del parto, lo que hace pensar que era importante.

Estas pacientes han tenido muchos hijos ( 5 en promedio) y pocos abortos (1.9 en promedio).

Un 22.5 por ciento informó que no estaba empleando ningún método anticonceptivo previo. Hay casos como el de Bangladesh en los que esta cifra llega al 74 por ciento. En nuestro estudio había además otro $16.1 \%$ de mujeres que empleaban métodos menos eficaces, como el del ritmo, espumas, jaleas, etc., para un total de $38.6 \%$, que o no usaban anticonceptivos o planificaban su familia con los métodos menos eficaces.
La mayoría, $55.9 \%$, estaba empleando anticonceptivos orales o dispositivos intrauterinos.

La obesidad extrema, las laparatomías previas múltiples, han sido consideradas contraindicaciones relativas de la laparoscopia. En nuestro estudio solamente hubo 4 pacientes de $80 \mathrm{~kg}$ o más y $10.6 \%$ tenían alguna cirugía abdominal previa.

Fueron por orden de frecuencia, cesáreas, colecistectomías, apendicectomía, herniorrafias y otras laparotomías por causas menos comunes.

La mayoría de las pacientes fueron mujeres sanas $194.1 \%$ no habian padecido enfermedad sistémica y $80 \%$ tenían al examen laparoscópico o de minilaparotomía un aparato genital interno normal).

En $20 \%$ se encontró alguna patología genital, como adherencias, miomatosis uterina, quistes ováricos, varicocele pélvico, etc.

Sólo en un $8.5 \%$ se consideró que había signos de infección pélvica crónica.

No hemos hecho una evaluación de cómo las pacientes consideran el dolor o las molestias experimentadas durante el procedimiento. En una investigación de este tipo, Penfield en 200 minilaparotomías, con anestesia local, encontró que el $29 \%$ de las mujeres habían experimentado dolor severo o moderado; sin embargo, sólo el $8 \%$ preferiría que le hubieran administrado anestesia general (6).

La práctica más común consiste en el empleo de anestesia local (2, 7, 8, 9, 10). En nuestro estudio ésto se hizo en el $77.5 \%$ de los casos. Las minilaparotomías se efectuaron casi siempre con anestesia general. 
En cuanto a complicaciones, registramos un $10.7 \%$ debidas al procedimiento y $5.6 \%$ a la esterilización en sí. Muchas de ellas, como se ha anotado en la literatura, ocurrieron al comienzo del programa, cuando nuestra experiencia era muy limitada. Ocurren también durante el entrenamiento de nuevos médicos residentes en la técnica correspondiente.

Hay complicaciones inmediatas, tales como: hemorragias, traumatismos, quemaduras, infecciones, dolor, complicaciones cardiorespiratorias por anestesia y neumoperitoneo, imposibilidad de realizar la esterilización. Estas son bien conocidas y ocurren prácticamente en todas las series publicadas.

Hay complicaciones a largo plazo, que son menos conocidas, pero que constituyen motivo de preocupación $y$ de estudio, tales como embarazos ectópicos subsiguientes $(11,13)$, al teraciones del ciclo menstrual $(12,14)$, problemas sépticos tardíos (15), dificultad mayor o menor para reversión mediante microcirugía tubaria (16), efectos sicológi$\cos (1)$.

Nuestros resultados sólo permiten obtener limitada información sobre las complicaciones inmediatas y ninguna sobre las complicaciones a largo plazo, por falta de control.

Las primeras, se presentaron casi exclusivamente en electrocoagulaciones laparoscópicas y no en minilaparotomías. Consistieron en orden de frecuencia en hemorragias, leves por lo general, perforaciones uterinas, dificultades técnicas para hacer el neumoperitoneo, en ocasiones por mal estado del equipo, quemaduras leves de pared adbominal o de órganos pélvicos, una falla para electrocoagular una trompa y otras de menor importancia.

No es posible sacar ninguna conclusión sobre índice de fallas de los méto- dos, por falta de control sistemático de las pacientes a largo plazo. Este es un serio problema en la mayoría de nuestros estudios clínicos.

Las complicaciones de la anestesia fueron en general leves $y$ sin consecuencia para las pacientes.

El perfeccionamiento de los equipos endoscópicos, hace que cada día sea más aceptable, segura y eficaz, la esterilización laparoscópica

Información básica sobre estos avances puede encontrarse en la literatura (17). Gracias a ellos se amplían cada vez más los horizontes de las aplicaciones endoscópicas. Están apareciendo nuevos y más amplios informes sobre esterilización laparoscópica puerperal y postaborto, con índices de morbilidad comparables a los del procedimiento empleado en período de intervalo $(4,(18,19)$.

Las quemaduras eléctricas, como complicación importante del uso de corrientes de alta frecuencia en sistemas monopolares, son suficientemente conocidas. Para evitarlas se han ideado los equipos bipolares, muy eficaces para ese propósito, pero que carecen, infortunadamente, de varias ventajas importantes de los sistemas monopolares (17). A consecuencia de ésto ofrecen mayor posibilidad de hemorragias (20).

Otra solución, que se viene empleando aproximadamente desde 1975, es la termocoagulación, usando corriente continua de bajo voltaje. También llamada endocoagulación, término menos significativo, más genérico. Tiene las siguientes ventajas: (a) la corriente eléctrica no entra en contacto con la paciente (b) la temperatura es preseleccionable de acuerdo con el tejido que se va a tratar (c) controlada visual y acústicamente (d) cohibe eficazmente las hemorragias y (e) se afirma que no produce adherencias postoperatorias $(17,21,22)$. 
Llama la atención en nuestro estudio el poco uso que se hizo, relativamente, de la minilaparotomía-Pomeroy $(13 \%$ de los casos). Esta última técnica, descrita inicialmente por Uchida en 1961, es más económica, sencilla y asociada a menor número de complicaciones que la electrocoagulación laparoscópica. Si se tienen en cuenta estos factores y las condiciones del medio colombiano, resulta altamente recomendable.

La prestación de servicios eficientes de laparoscopia requiere mayores iriver siones en repuestos y mantenimiento, así como también la adquisición de nuevos equipos que representen mejoras reales y significativas desde el punto de vista técnico.

No obstante lo anterior, se prevé que para 1985 se habrá duplicado, con respecto a 1978 , vel número dè parejas que usen la anticoncepción quirúrgica voluntaria en el mundo (1).

\section{Resumen}

Se estudian 460 pacientes esterilizadas por laparoscopia-electrocoagulación y 69 por minilaparotomía-Pomeroy.

Se describen las características más sobresalientes de las usuarias y las complicaciones inmediatas de los procedimientos.

Se comparan nuestros resultados, con algunos de los encontrados en la literatura.

Se destaca la importancia de progresos técnicos, como el de los sistemas bipolares con corriente de alta frecuencia y el de la termocoagulación (endocoagulación).

Se insiste en las ventajas que la minilaparotomía tiene sobre la electrocoagulación laparoscópica en el medio colombiano y la conveniencia de fomentar su uso.

\section{FEMALE STERILIZATION}

\section{Summary}

This study was done with 460 patients sterilized by laparoscopy-electrocoagulation, and 69 by minilaparotomy-Pomeroy.

The article gives the most relevant characteristics of patients and describes the inmediate complications after the procedure. Some of these results are compared with results of other studies in especialized literature.

\section{Bibliografía}

1. Esterilización Voluntaria: El Método Anticonceptivo de Mayor Aceptación en el Mundo. Population Reports. Tópicos Especiales. No. 2 octubre de 1978.
The importance of technical progress should be noted, particularly in relation with bipolar systems of high frenquency electric current, and thermocoagulation (endocoagulation) systems.

In the Colombian context, minilaparotomy appears to be more useful than laparoscopy-electrocoagulation. Therefore the use of this technique must be promoted.

2. Female Sterilization by Mini-Laparotomy. Population Reports. Series C Number 5. November 1974.

3. Esterilización Tubaria. Revisión de Métodos. Population Reports. Serie C. Número 7. Diciembre 1977. 
4. BECK P., GAL D: Silicone Band Technique for Laparoscopic Tubal Sterilization in the Gravid and Nongravid Patient. Obstet. Gynecol 53: 653, 1979.

5. BECK P., GAL D. TANGER L: Silicone Band Sterilization with Radiographic and Laparoscopic Evaluation. Obstet Gynecol 53:698, 1979.

6. PENFIELD AJ: Minilaparotomy for female Sterilization. Obstet. Gynecol 54: $184,1979$.

7. La Esterilización por Laparoscopia. Una Nueva Técnica. Informes Médicos. Serie C. Número 1. Enero 1973.

8. Esterilización Laparoscópica con Clips. Informes Médicos. Serie C. Número 4. Junio 1977.

9. METHA PV: Laparoscopic Stertlization with the Falope Ring: Experience with 10.100 Women in Rural Camps. Obstet Gynecol 57:345, 1981 .

10. GUZMAN R. Y COL: Una evaluación de la esterilización por laparoscopia consecutiva al aborto incompleto. Rev. Col. Obstet. Gynecol 30:57, 1979.

11. CUNANAN RG, COUREY NG, LIPPES $\mathrm{J}$ : Complications of Laparoscopic Tubal Sterilization. Obstet Gynecol 55:501, 1980.

12. BAGGISH MS: Complications of Laparoscopic Sterilization. Comparison of Two Methods. Obstet Gynecol 54:54, 1979.

13. WOLF GC, THOMPSON NJ: Female Sterilization and Subsequent Ectopic Pregnancy. Obstet Gynecol 55:17, 1980.
14. DONNEZ J: Luteal Function After Tubal Sterilization. Obstet. Gynecol 67:65, 1981.

15. GLEW RH, POKOLY TB: Tuboovarian Abscess Following Laparoscopic Sterilization with Silicone Rubber Bands Obstet Gynecol 55:760, 1980.

16. Recuperación Quirúrgica de la Fecundidad después de Esterilización. Population Reports. Serie C. Número 8. Julio 1981.

17. Guía para la Selección de Equipo para Procedimientos de Esterilización M/F. Population Reports. Serie M. Número 1. Febrero 1980.

18. McDONELL CF: Puerperal Laparoscopic Sterilization. Am. J. Obstet. Gynecol 137:910, 1980.

19. Esterilización Laparoscópica II. ¿Cuales son los Problemas? Informes Médicos. Serie C. Número 2. Marzo 1973.

20. POUS-IVERN IC: La esterilización tubárica en laparoscopia con corriente monopolar. Obstet. Gynecol Latinoamericana 36:156, 1978.

21. GUNNING JE. TOMASULO JP. GARITE TH: Laparoscopic Tubal Sterilization Using Thermal Coagulation. Obstet Gynecol 54:505, 1979.

22. MILLAS N. BAGNATI E: La endocoagulación en la cirugía laparoscópica ginecológica. Obstet Ginecol Latinoamericana $36: 32,1978$. 